\title{
Vessel Architecture and Excessive Coronary Artery Instrumentation as a Cause of Coronary Artery Perforation
}

\author{
Chiung-Jen $\mathrm{Wu}^{1}$, Ahmad Mohammad Alkonaissi ${ }^{2 *}$ and Cheng-Jui Lin ${ }^{1}$ \\ ${ }^{1}$ Department of Internal Medicine, Chang Gung University School of Medicine, Kaohsiung Chang Gung Memorial Hospital Medical Center (KCGMH), \\ Kaohsiung, Taiwan \\ ${ }^{2}$ Cardiology Department, National Heart Institute, Egypt
}

Submission: February 05, 2017; Published: March 02, 2017

*Corresponding author: Ahmad Mohammad Alkonaissi, 24 July $26^{\text {th }}$ street, Lebanon square, Almohandessin city, Giza governorate, Egypt, Tel: +201001851190; Email: Ahmadalkonaissi@gmail.com

\begin{abstract}
Coronary artery perforation (CP) is a rare but catastrophic complication of percutaneous coronary intervention (PCI). Grade three coronary artery perforation and rupture results in pericardial effusion and tamponade requiring urgent pericardiocentesis. Advances in coronary intervention have increased the opportunity to treat coronary artery perforation. We report a case of an elderly male patient with multiple cardiovascular risk factors presenting with Non-ST Elevation Myocardial Infarction (NSTEMI) complicated with pulmonary edema. Coronary angiogram (CAG) revealed severe triple vessel coronary artery disease (CAD) with heavy calcifications. Successful recanalization of mid left anterior descending coronary artery (LAD) chronic total occlusion (CTO) with heavy and floppy guidewires followed by stepwise rotational atherectomy (RA) with two different sizes burrs and high pressure balloon (HPB) dilation then deploying three drug-eluting stents to proximal to very distal (LAD), with post-dilation with (HPB) then deploying drug-eluting stent to tortuous proximal to mid left circumflex. After PCI, Grade three coronary artery perforations was noted at the stented mid to distal (LAD) acute cardiac tamponade developed to which emergent prolonged balloon inflation, reversal of anticoagulation and pericardiocentesis were done, covered stents were deployed.
\end{abstract}

Keywords: Coronary artery perforation; Percutaneous coronary intervention; Chronic total occlusion; Non ST-segment myocardial infarction; Rotational atherectomy; High pressure balloon

\section{Introduction}

Coronary artery perforation (CP) is a rare but catastrophic complication of percutaneous coronary intervention (PCI). Perforation occurs in $0.1 \%$ to $3 \%$ of cases with mortality rates approximately $10 \%$. Grade III coronary perforation and rupture results in pericardial effusion and tamponade requiring urgent pericardiocentesis in up to $60 \%$ of cases [1]. Advances in coronary intervention have increased the opportunity to treat coronary artery perforation. We report a case of 87 years old male with multiple cardiovascular risk factors who presented with non-ST segment elevation myocardial infarction (NSTEMI) complicated by pulmonary edema. Coronary angiogram (CAG) revealed severe triple vessel coronary artery disease (CAD) with heavy calcifications of the left main coronary artery and its branches. During (PCI), he had Ellis type III coronary artery perforation, cardiac tamponade and shock. The patient was supported with inotropes and intra-aortic balloon pump (IABP), prolonged balloon inflation was done, pericardiocentesis was done, and covered stents were deployed.

\section{Case Report Information, CAG and PCI}

The patient was 87 years old male with a history of hypertension, type two diabetes mellitus presenting with shortness of breath progressively ending into respiratory distress over two days before admission to our department, the patient had a history of chronic kidney disease stage 3 , serum creatinine was $1.68 \mathrm{mg} / \mathrm{dl}$ on admission which became $2.23 \mathrm{mg} / \mathrm{dL}$ thereafter, and positive Toponin I at admission, electrocardiogram showed sinus tachycardia with minimal ST depression at inferolateral leads, minimal ST segment elevation at V1-V4 with pathological Q waves, chest $\mathrm{x}$-ray showed pulmonary edema, under impression of NSTEMI he was admitted and scheduled for an angiogram which showed severe triple vessel coronary artery disease with heavy calcifications of left system as illustrated in Figures 1 \& 2; the proximal left anterior descending artery (LAD) had severe stenosis 93\%, the mid left anterior descending artery (LAD) had chronic total occlusion 
(CTO) after giving a large septal branch and second diagonal (D2), collaterals to CTO segment from distal left circumflex artery (LCX) Grade 1/3; p-LAD bridging collaterals Grade 1/3 and large epicardial tortuous collateral from conus branch to distal LAD Grade 3/3, the proximal left circumflex artery (LCx) was acutely angled tortuous vessel with heavy calcifications showing proximal stenosis $74 \%$, and the right coronary artery (RCA) was small non-dominant diffusely diseased vessel (Syntax score: 39.5), Echocardiogram showed borderline left ventricular performance with resting wall motion abnormalities, dyspnea then progressed, and acute respiratory distress developed that necessitated respiratory support with mechanical ventilation and hemodynamic support with intra-aortic balloon pump (IABP).

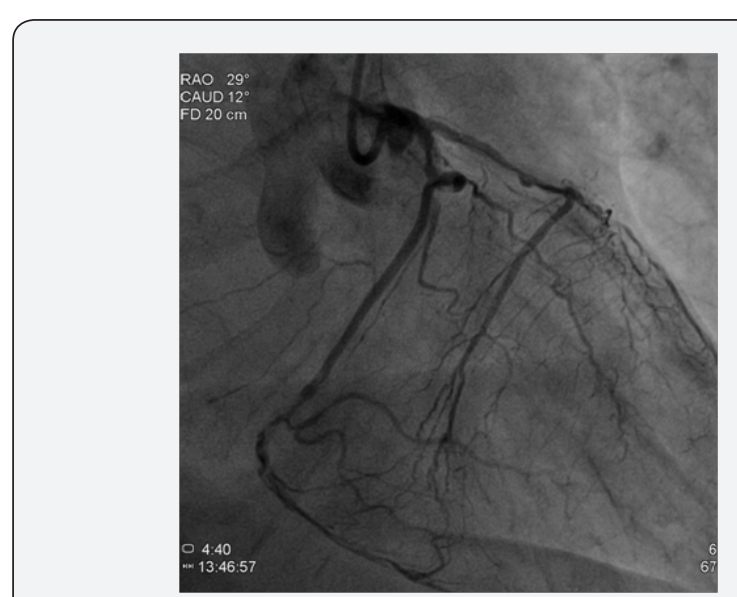

Figure 1: Coronary angiogram of the left main, left anterior descending and left circumflex arteries.

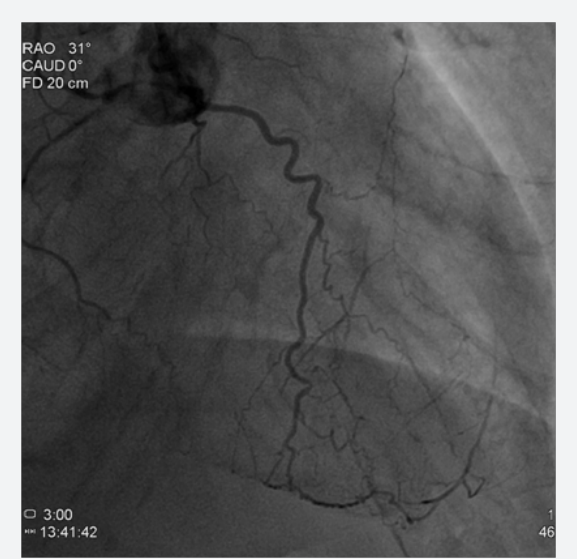

Figure 2: Coronary angiogram of the right coronary artery and conus artery.

In order to perform (PCI) left trans-radial (TR) access was established then with the use of sheath-less technique seven French system (7F EBU4) (Launcher Medtronic) guiding catheter (GC) was used to engage the left main coronary artery (LMCA). A 0.014 Fielder FC guidewire (GW) was loaded on single lumen micro-catheter (MC) and was used to re-canalize CTO from mid LAD. After failure to do so using two Fielder FC guidewires, we switched to a Fielder XT-A guidewire which was successful to pass to the severely calcified mid to distal LAD with ante-grade approach. With anchoring balloon technique a $2.0 \times 20 \mathrm{~mm}$ Mini-Trek balloon at D2 branch at 12 atmospheric pressure unites (atm), we inflated a $1.2 \times 6 \mathrm{~mm}$ Mini-Trek(Abbott Vascular) balloon at 16-20atm at the CTO cap, then 2.0x20 MiniTrek balloon at mid to distal LAD at 18-20 atm. Long dissection from very distal LAD was noted. To facilitate delivery of stents a 0.009 " Rota Wire was substituted for Fielder FC to very distal LAD and rotational atherectomy (RA) de-bulking was done in a stepwise manner with $1.25 \mathrm{~mm}$, then $1.5 \mathrm{~mm}$ burr at $180 \mathrm{~K}-220 \mathrm{~K}$ rpm as illustrated in Figures $3 \& 4$. We dilated the whole LAD with $2.0 \times 20 \mathrm{~mm}$ Mini-Trek at 20 atm then $2.5 \times 15 \mathrm{~mm}$ Hiryu (TERUMO) high pressure balloon (HBP) at $28 \mathrm{~atm}$ at proximal to mid LAD, then $3.0 \times 15 \mathrm{~mm}$ Hiryu HPB was inflated at p-mLAD at 24-26 atm in order to facilitate stent passage through calcified mid LAD, subsequently we interrogated the LAD with intravascular ultrasound (IVUS) as illustrated at Figure 5.

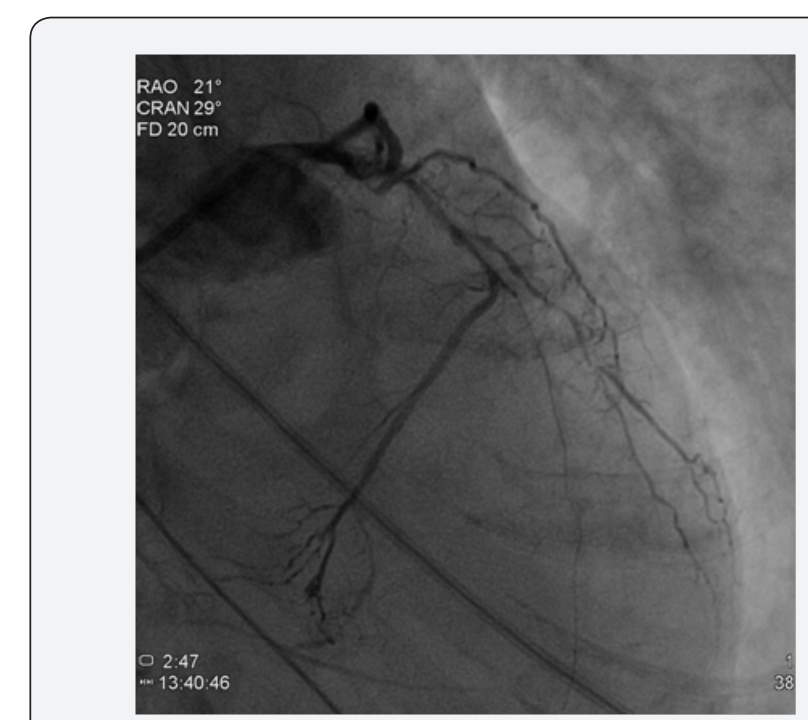

Figure 3: Re-occlusion of the $L A D$ after recanalization.

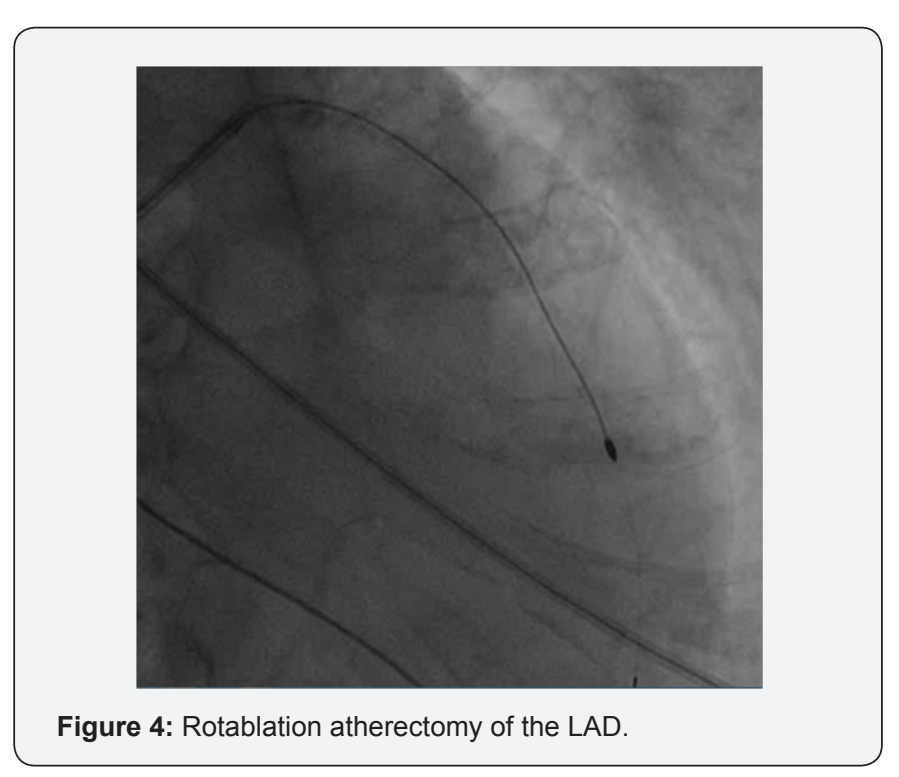




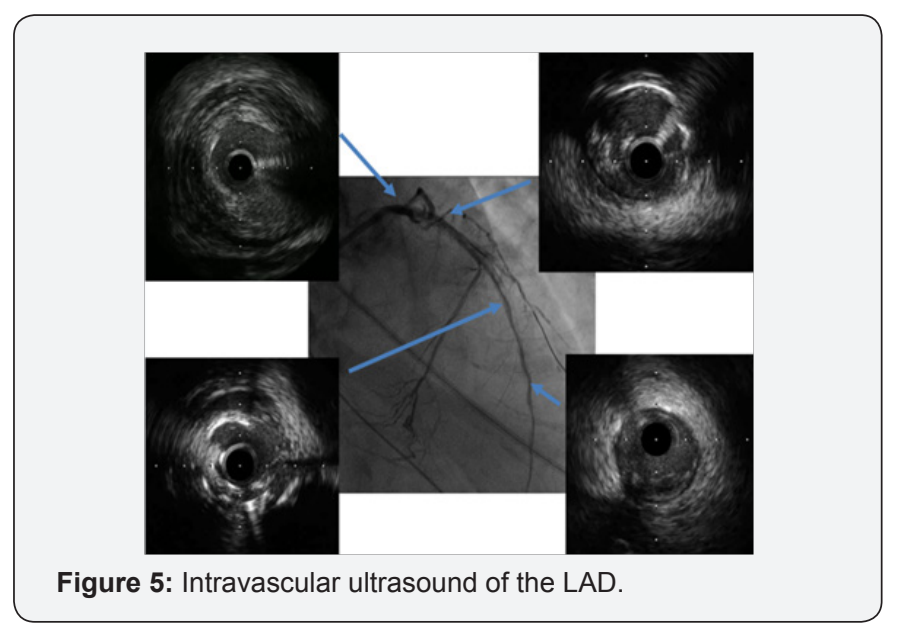

Three drug eluting stents (DES) everolimus eluting stents (EES) Xience Prime $2.25 \times 28 \mathrm{~mm}, 2.75 \times 38 \mathrm{~mm}$, and $3.0 \times 38 \mathrm{~mm}$ (Abbott Vascular) were deployed from very distal to proximal LAD at 7-10atm, and followed by post-dilation with a $3.0 \times 15 \mathrm{~mm}$ Hiryu (TERUMO) HPB at mid to proximal LAD at 20-24atm. A new Fielder FC GW was loaded on a second new Fine-cross MC and was advanced to distal LCx in order to treat the proximal LCX stenosis. With anchoring a 3.0x15mm Hiryu (TERUMO) HPB at mid LAD at 20atm, a 1.2x6mm Mini-Trek balloon (Abbott Vascular) was passed through acute-angled proximal to mid LCx, then inflated at mid LCx at 20atm, then further dilatation with $2.0 \times 12 \mathrm{~mm}$ Mini-Trek balloon (Abbott Vascular) at 20atm and $2.5 \times 15 \mathrm{~mm}$ Hiryu (TERUMO) HPB at 26atm.

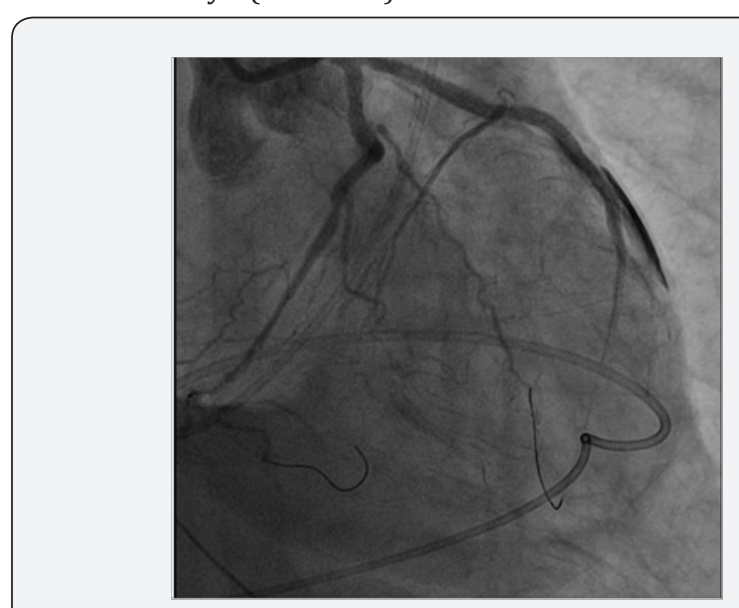

Figure 6: Ellis type III coronary artery perforation of the LAD.

Ellis type III perforation from stented mid to distal LAD was noted as illustrated in Figure 6. Initially we used balloon inflation at the perforation site to seal the leakage. Bedside 2 Dimensional echocardiography showed acute cardiac tamponade with surrounding effusion of more than 100cc. Emergent pericardocentesis was done via subcostal approach with systolic blood pressure restored to more than $100 \mathrm{mmHg}$ after draining bloody effusion $(250 \mathrm{cc}$ ) from pig-tail catheter. Protamine $50 \mathrm{mg}$ was administered. We completed deployment of a $2.75 \times 33 \mathrm{~mm}$ (EES) Xience Prime DES (Abbott Vascular) at proximal to mid
LCx under 5.5F guide-liner catheter support and anchoring a $3.0 \times 15 \mathrm{~mm}$ Hiryu (TERUMO) HPB at mid LAD at 24atm.

A 2.8x19mm polytetrafluoroethylene (PTFE) covered stent Graft-master stent (Abbott Vascular) was placed at mid to distal LAD at 16 atm followed by post-dilatation with 3.0x15mm Hiryu (TERUMO) HPB at 26 atm. Persistent dye extravasation was noted. Another $2.8 \times 16 \mathrm{~mm}$ Graft-master covered stent was placed at distal LAD overlapped the last one, sealing the perforation site as illustrated in Figure 7. The patient gradually improved under ventilator \& IABP support.

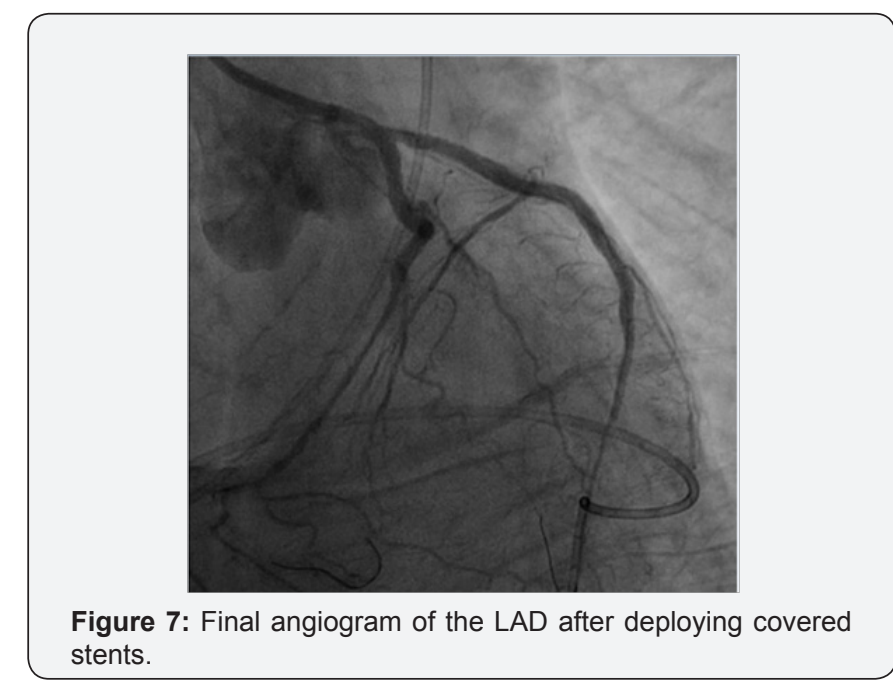

\section{Discussion}

Percutaneous coronary intervention has become a leading therapeutic treatment option for coronary artery disease, especially in the setting of ACS [2]. Chronic total occlusions (CTO) remain the most challenging lesion subset in percutaneous coronary interventions (PCI). This is due to the suboptimal visualization of the vessel distal to the occlusion (exclusively through collaterals) and the adverse anatomical characteristics of the plaque, which is usually resistant to wire manipulation due to fibrotic and calcific components. Conventional CTO wiring techniques (antegrade "true-to-true lumen" approach) have traditionally been associated with low success rates (60$80 \%$ ) and higher incidence of complications, as compared with non-CTO intervention [3], modern practice have shown more wide adoption of sub-adventitial techniques for CTO PCI.

In our case here patient related risk factors for CP where, hypertension, elderly age, chronic renal insufficiency, coronary artery calcification, tortuosity, and type C lesions, while procedure related risks include the use of hydrophilic wires, IVUS and Rotational atherecromy, and high pressure balloon inflation either for post-dilation or for anchoring. In a metanalysis of 16 studies by Shimony et al. [4] involving 197, 061 PCIs, the pooled incidence of CP was $0.43 \%$ (95\% CI 0.35-0.52\%) [4].

According to Al-Lamee et al. [2] study of the incidence and the outcome for grade III CP, the commonest cause was inflation 
of an intracoronary balloon, with no change in the incidence based on the balloon compliance. Grade III CP was caused by rotablation, directional atherectomy, and cutting balloon inflation in only a small number of cases [2].

In a cohort conducted by Barnett et al. [1] the acute and up to 12 month outcome of 6031 PCI from 2005 through 2011, patients who received PFTE covered stents were followed to evaluate the incidence of major cardiac events. Major cardiac events were defined as: cardiac tamponade, death, emergent surgical drainage, myocardial infarction, or the need for emergent coronary artery bypass grafting, PFTE graft stent placement for coronary artery perforation during PCI was found to be beneficial acutely and up to 12 months following PFTE placement [5].

In 2005, Colombo et al. [6] suggested an approach for the recanalization of occluded coronary arteries, which was termed "Subintimal Tracking and Re-entry" (STAR technique). STAR demonstrated that dissecting through the sub-adventitial space could traverse long occlusion segments safely and rapidly, increasing operators' confidence in traversing this blind end segment. Briefly, STAR creates a sub-adventitial cleavage plane by advancing a hydrophilic coronary wire with a J-loop configuration at its tip ("knuckle") to allow a blunt dissection between the anatomical planes of the vessel, with the aim to re-enter into the true lumen distal to the occlusion. Initial experience with STAR was encouraging, with final thrombolysis in myocardial infarction (TIMI) 3 flow achieved in $97 \%$ of cases, and full recanalization of the main vessel and most distal branches in $67 \%$. Coronary artery perforations were observed in $10 \%$ of patients. Five-month follow-up showed restenosis rates of $(52 \%)$, initially attributed to the use of bare-metal stents (BMS), first-generation drug-eluting stents (DES), and long stented segments [6]. TIMI flow grade $<3$ following stenting independently predicted restenosis [7].

\section{Conclusion}

CTO PCI has been associated with a variety of complications of these $\mathrm{CP}$ is the most feared and dramatic life-threatening complication. All operators, assistants and technicians should be prepared with required skills, technology and tools to avoid and manage such catastrophic complication. The use PTFE covered stents is considered the most optimal available therapeutic option for acute management of CP especially with unfavorable hemodynamics and persistent leakage.

\section{References}

1. Barnett BC, Berrio G, DS Gantt (2014) Outcomes Of Pfte Covered Stents Implanted During Pci For Management Of Acute Coronary Perforation. Allschwilerstrasse, Karger, Switzerland.

2. Al-Lamee R, Lelasi A, Latib A, Godino C, Ferraro M, et al. (2011) Incidence, Predictors, Management, Immediate and Long-Term Outcomes Following Grade III Coronary Perforation. JACC Cardiovasc Interv 4(1): 87-95.

3. Sumitsuji S, Inoue K, Ochiai M, Tsuchikane E, Ikeno F (2011) Fundamental Wire Technique and Current Standard Strategy of Percutaneous Intervention For Chronic Total Occlusion With Histopathological Insights. JACC Cardiovasc Interv 4(9): 941-951.

4. Shimony A, Joseph L, Mottillo S, Eisenberq MJ (2011) Coronary Artery Perforation During Percutaneous Coronary Intervention: A Systematic Review And Meta-Analysis. Can J Cardiol 27(6): 843-850.

5. Brilakis ES, Banerjee S, Karmpaliotis D, Lombardi WL, Tsai TT, et al. (2015) Procedural Outcomes of Chronic Total Occlusion Percutaneous Coronary Intervention: A Report from the NCDR (National Cardiovascular Data Registry). JACC Cardiovasc Interve 8(2): 245-253.

6. Colombo A, Mikhail GW, Michev I, Iakovou I, Airoldi F, et al. (2005) Treating Chronic Total Occlusions Using Sub-Intimal Tracking and Reentry: The STAR Technique. Catheter and Cardiovas Interv 64(4): 407-411.

7. Katritsis DG, Meier B (2008) Percutaneous Coronary Intervention For Stable Coronary Artery Disease. Journal of the American College Cardiology 52(11): 889-893.

8. Carlino M, Figini F, Ruparelia N, Uretsky BF, Godino C, et al. (2016) Predictors Of Restenosis Following Contemporary Subintimal Tracking and Reentry Technique: The Importance of Final TIMI Flow Grade. Catheter Cardiovasc Interv 87(5): 884-892.

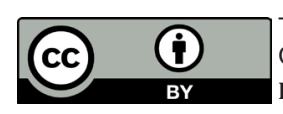

This work is licensed under Creative Commons Attribution 4.0 License DOI: $10.19080 /$ JOCCT.2017.03.555612

\section{Your next submission with Juniper Publishers} will reach you the below assets

- Quality Editorial service

- Swift Peer Review

- Reprints availability

- E-prints Service

- Manuscript Podcast for convenient understanding

- Global attainment for your research

- Manuscript accessibility in different formats

( Pdf, E-pub, Full Text, Audio)

- Unceasing customer service

Track the below URL for one-step submission https://juniperpublishers.com/online-submission.php 